\title{
Social Risk Factors for Asthma Exacerbations Among Pediatric Patients: A Scoping Review
}

\author{
Shriya Vinjimoor ${ }^{1}$, Alex Meltzer², Eneida Mendonca ${ }^{2}$ \\ ${ }^{1}$ Indiana University School of Medicine, ${ }^{2}$ Regenstrief Center for Biomedical Informatics
}

\begin{abstract}
Background: Asthma is the most common chronic illness among the pediatric patient population. While several studies have investigated the biological and environmental factors associated with asthma exacerbations, research on the relationship between asthma and social determinants of health is limited. This scoping review addresses this gap in knowledge by exploring social factors that are associated with the risk of asthma exacerbations among pediatric patients and mapping identified variables onto the SAVI database.
\end{abstract}

Methods: The OVID Medline, Embase, and Psyclnfo databases were systematically searched for studies published as of July 2, 2021. No restrictions on study design or publication type were made but search was limited to English manuscripts. Resulting studies were screened based on phased eligibility criteria on Covidence. In Phase 1, duplicates were removed. In Phase 2, studies unrelated to asthma and those with participants outside of 2-18 years of age were excluded based on title and abstract screening. In Phase 3, studies that investigated asthma exacerbations as well as its association with social factors were retained while studies solely looking at non-social factors or abstract-only studies were excluded based on the full text screening. In Phase 4, data on frequency of both inpatient and outpatient physician visits for asthma were extracted along with information on study characteristics and social factors.

Results: Following the initial database search, 3312 studies were retrieved for the scoping review. 772 duplicates were removed during Phase 1. 1628 studies were excluded during the abstract and title screening in Phase 2. Upon resolving 384 conflicts, the remaining 912 studies proceeded to full text screening in Phase 3.

Conclusion: The initial literature screening suggests an association between social risk factors and pediatric asthma exacerbations. Incorporating large scale change of these social factors with individual pharmacological treatment could help improve pediatric asthma outcomes. 